\title{
IMPORTANCIA DE LOS COSTOS DE LA CALIDAD Y NO CALIDAD EN LAS EMPRESAS DE SALUD COMO HERRAMIENTA DE GESTIÓN PARA LA COMPETITIVIDAD
}

\section{RESUMaN}

$\boldsymbol{E}$ l objetivo de este artículo es analizar la importancia que tiene la medición sistemática de los costos de inversión en calidad y aquellos injustificados por fallas en los procesos de la empresa, con el fin de estimular en los directivos el interés por conocer su monto para que la gerencia tenga bases cuantitativas para la toma de decisiones, en lo que respecta a la contención de los costos de operación y evalúe mes a mes su impacto.

Una decisión eficaz, debe reducir directamente los costos de no calidad en el proceso. Se realiza una revisión bibliográfica de los temas seleccionados y se exponen los más representativos factores que influyen en los mayores costos injustificados considerados de no calidad. Como conclusión relevante, la multiplicidad de procesos, procedimientos y normas de las empresas de salud, hacen muy importante que la organización tenga establecidos métodos para la cuantificación de las fallas, errores y repeticiones, y así contar con bases objetivas para la toma de decisiones sobre la contención de dichos costos.

\section{Palabras Clave}

Costos de calidad

Costos de no calidad
Luis Javier Arango Cardona*

\section{Introducción}

\section{La reducción significativa} de los techos financieros ante los cuales operan las organizaciones de salud en el Sistema General de Seguridad Social en Salud, con unos costos administrativos fijados por la legislación en un máximo del $10 \%$ y un control de los copagos o pagos compartidos del usuario por la misma legislación, sumada al reconocimiento de las condiciones especiales de funcionamiento de este tipo de empresas, con un alto riesgo financiero generado por el difícil control del gasto, hace imperativo el reconocimiento de las actividades y costos que no agregan valor, con el fin controlarlos y garantizar la sostenibilidad económica y su permanencia en el mercado.

Médico cirujano. Magister en Salud Pública AAMH Docente especialización Hospitalaria EAN. Creador de un Modelo de Sistema de Medición de Costos de Calidad y No calidad para Empresas de Salud en implementación en organizaciones de salud de mediana y alta complejidad en Bogotá D.C. Colombia. 
Ante la evidencia mundial documentada sobre la relevancia de los costos de inversión en calidad y los producidos por las fallas en la multiplicidad de procesos existentes en una empresa de salud, conocidos como Costos de No Calidad, injustificados y significativamente altos en las empresas en las cuales han sido cuantificados, se ha venido considerando cada vez con mayor importancia, la medición y análisis de estos costos en las organizaciones de salud, con el fin de apoyar la toma de decisiones de mejoramiento y control, en la adopción de estrategias de contención de costos, que logren garantizar la viabilidad financiera y la obtención de excedentes operativos para su desarrollo científico y tecnológico.

Se analizan en el presente artículo, las variables más reconocidas para el cálculo de los costos de inversión en calidad de los procesos y las causas más frecuentes de fallas en los mismos (No Calidad). Sin querer ser exhaustivo, la relación de ellas, busca ampliar la visión de los directivos de las empresas de salud sobre la alta probabilidad de ocurrencia de fallas, generando costos de operación que pueden reducirse.

La revisión bibliográfica analizada, demuestra el interés y la importancia que le dan al tema, tratadistas de renombre internacional en Calidad Total; el reconocimiento de estos costos permite apoyar las decisiones de mejoramiento y control.

\section{Abstract}

This paper aims at analyzing the importance of cost systematic mediation in quality investment and those unjustified as the result of company process failure, with the purpose of motivating the directive staff to know the total amount they have, allowing managers to have quantitative bases for decision talking in respect with the contention of operational costs and to evaluate monthly. The impact of such decisions: one efficient decision may directly reduce the nonquality cost in this process. A bibliographic revision is made on selected topics and the most representative factors that influence the major unjustified costs considered as nonquality. Concluding, the variety in processes, procedures and norms of health companies makes them have established methods to quantify failures, errors and repetitions to count on objective fundamentals to make decisions on the contention of such costs.

The author's experience 1 for more than 25 years on health programs and companies of medium and high complexity and the fact that he is the creator of a cost measuring system of quality and non- quality, being under the process of implementation in some health centers in Bogota city, whose results are about to be published, allowing the presentation of objective and integral motives for the fulfillment of the main objective of this paper, which the motivation of health company managers are for measuring such costs to establish the most effective contention strategies to guarantee the financial sustainability of this kind of organization.

\section{KEY Words}

Quality costs Non- quality costs 


\section{2. Овлетіvo}

Destacar la importancia del conocimiento sistemático y confiable de los costos invertidos en la calidad y aquellos no justificados o de No Calidad de los procesos en las empresas, como una herramienta de gestión en las empresas de salud, para la toma de decisiones basada en resultados cuantitativos, que permitan así mismo, la evaluación del impacto de las medidas de control o mejoramiento al respecto.

\section{INVERSIÓN EN CALIDAD}

$S_{i}$ bien es cierto, la publicidad y presentación de un producto o servicio puede lograr su penetración al mercado y la obtención por la empresa de importantes utilidades, solo la calidad de esos servicios oproductos permitirán que los clientes permanezcan y continúen fieles a la empresa.

La no calidad es detectada rápidamente por los clientes y ocasiona su retirada y malestar, situación alarmante para cualquier organización que quiere permanecer en el mercado, por la dificultad para atraer nuevamente a aquellos que se alejan ante el reconocimiento de problemas de calidad en los productos 0 servicios de determinada organización.
La inversión para la calidad, está determinada por todos los recursos dedicados a una serie de actividades que buscan prevenir que ocurran errores o fallas en el proceso, por ejemplo en la facturación; estos se denominan costos de prevención, como:

- Diseño y rediseño de procesos.

- Capacitación para la implementación y ejecución de los procesos.

- Reuniones para evaluación de funcionamiento y mejoramiento de procesos.

- La inducción y la reinducción.

También considerados como inversión en calidad, son aquellos recursos dedicados a la auditoría y control de cada proceso, por ejemplo:

- La auditoría y el control de la facturación.

- La revisión y auditoría de cuentas.

Todas las anteriores actividades realizadas con el fin de: diseñar, rediseñar, capacitar y realizar el seguimiento y control por ejemplo de la facturación, las cuentas, la contratación, la gestión de cartera, la atención de urgencias, el laboratorio clínico y la radiología para garantizar que los resultados de cada proceso se logren conforme fue programado, se constituyen como acciones de prevención y evaluación de la calidad. 
Estos dineros invertidos en el mantenimiento y mejoramiento de los procesos, se consideran inversión en calidad, es decir, en esfuerzos para que se logre una atención de los clientes con la mayor eficiencia. Se invierten para lograr la mejor calidad, costos que no son evidentes mes a mes ni siquiera para los directivos, porque no se tienen en la mayoría de las empresas, mecanismos para calcular lo que se invierte cada mes en la calidad de los procesos.

Obviamente, no puede conocerse mensualmente si dichos costos se incrementan o disminuyen, si corresponden a los requerimientos reales para que cada proceso logre la calidad esperada, o si los costos son altos en forma innecesaria o injustificada.

Se invierte permanentemente para mantener y mejorar la calidad de cada proceso, pero:
- ¿Cuánto se invirtió en cada proceso?

- ¿En qué actividades?

- ¿Cuál es el monto considerado adecuado para que cada proceso logre la calidad esperada?

- ¿Cuándo intervenir o que decisiones tomar para reducir o incrementar la inversión en calidad?

- ¿Con qué soportes?

- ¿Cuál ha sido la inversión en calidad de cada proceso en los meses anteriores?

- ¿Qué proporción de esta inversión representa el costo del proceso?

- ¿Cuál es la composición del costo de la inversión realizada en calidad cada mes?

\section{4. ¿Cómo saber cuánto estamos PERDIENDO POR LA NO CALIDAD?}

\begin{abstract}
Tan importante o más que conocer los costos de la inversión en la calidad, es poder cuantificar en forma confiable, oportuna y sistemática, los gastos en que incurre la empresa por las fallas, repeticiones y errores en los procesos (Costos de No Calidad).
\end{abstract}

A pesar de que en forma permanente en cada proceso se realizan repeticiones por ejemplo por facturas objetadas por la auditoría porque no tienen los datos completos, no se codificaron los procedimientos en forma correcta o no se adjuntaron los soportes, nadie conoce cuántos recursos se invirtieron en la corrección y repetición, 
ni tampoco el monto en dinero perdido por tener que reprocesar la facturación para lograr pasarla en forma correcta para alcanzar el pago por los servicios prestados.

Si consideramos que no se trata de una sola factura, sino de cientos o miles, así como de múltiples repeticiones y fallas en cuentas, contratación, repeticiones de procedimientos $\mathrm{o}$ atenciones y otros procesos, el monto debe ser considerable y hace indispensable cuantificar e impactar el problema en forma positiva, para reducir al máximo estos costos injustificados. Con la multiplicidad de procesos existentes en una empresa de salud y las posibilidades de errores en cada uno, el dinero perdido por No Calidad, es considerable y por lo tanto, es imperativo conocerlos para poder establecer mecanismos para su control, con el fin de reducir los costos de operación, en busca de una mejor rentabilidad de la organización.

Estos errores o problemas en los procesos que ocasionan gastos injustificados se han denominado:

\subsection{Fallas internas}

Cuando los errores o fallas son detectadas por la misma empresa, antes de ser entregado el producto o servicio a los clientes. La probabilidad de ocurrencia de estas fallas en número significativo por la multiplicidad de procesos en ejecución y las características de operación de la mayor parte de las empresas, como la alta rotación de personal entre otras, determina que los montos en dinero perdido son importantes, en actividades como las siguientes:

- Repetición de la factura.

- Repetición de una cuenta.

- Repetición de una atención médica o una cirugía.

- Reproceso para corregir los soportes de la facturación en los servicios donde fue atendido el paciente.

- Repetición de exámenes de laboratorio clínico.

- Exámenes de laboratorio clínico que son realizados pero no se utilizan en el proceso asistencial del paciente, porque sus resultados salen cuando el paciente ya salió o se operó por ejemplo.

- Exámenes de radiología no reclamados por los pacientes o no utilizados en la atención del paciente.

- Atenciones repetidas por causas del paciente, la empresa o los prestadores.

- Internación prolongada por infección nosocomial.

Si bien es cierto, la corrección o repetición de estos procesos le cuesta a la empresa, el servicio o producto, 
puede entregarse al cliente en buenas condiciones.

\subsection{Fallas externas}

Fallas o errores de los procesos, que no son detectados en la empresa, sino que llevan a reclamaciones o glosas por los clientes, situación que puede ser incluso más alarmante, porque afecta seriamente la contratación próxima, el regreso del paciente a la empresa o la imagen corporativa, lo cual representa costos altos significativos, más representativos para el buen funcionamiento y la rentabilidad de la organización.

ejemplos:

Los siguientes son algunos

- Glosas de facturas y cuentas por errores en los códigos, nombres, tipo de eventos cobrados o falta de los soportes establecidos por ejemplo.

- Quejas por problemas en la atención de pacientes, presentada por la EPS.

- Reclamación de pacientes por mala atención.

- Requerimientos de la Superintendencia Nacional de Salud por fallas en la atención de salud.

- Cancelación de procedimientos o cirugías.
- Días de hospitalización producidos por infección nosocomial.

- Exámenes de laboratorio repetidos o no utilizados para la atención del paciente, no entregados debidamente antes del egreso hospitalario del paciente.

Todos estos problemas llevan a la empresa a invertir considerables recursos humanos, físicos, tecnológicos, científicos para resolverlos, dejando sin embargo a los clientes con la percepción de que tenemos debilidades y problemas de calidad.

Las consecuencias pueden ser más importantes y costosas que las ocasionadas por las fallas internas, porque adicionalmente pueden llegar a alterar la contratación futura o la demanda por nuevos clientes o lo que es peor, por los clientes actuales, no satisfechos con la atención recibida.

De mayor repercusión aún, es la consecuencia de las fallas en la atención, cuando se convierten en demandas judiciales y terminan en fallos de tutela o judiciales, con sanciones económicas a la entidad, en ocasiones, de importante monto en dinero, adicional a la pérdida de imagen y confianza que esto genera en los clientes.

Contar con un sistema de costos por proceso, permite conocer en forma sistemática, cuánto se invierte en la calidad de cada proceso y cuánto se gasta por las fallas en los mismos, 
lo cual dará una herramienta gerencial de gestión para la toma de decisiones de mejoramiento o control de costos y evaluar su impacto al mes siguiente.

Si se adopta una metodología estándar para la medición de dichos costos en forma sistemática y confiable, la inversión en calidad deberá reflejarse en la reducción de los costos de No Calidad.
Se estarán gerenciando los procesos hacia el mejoramiento, con decisiones específicas de control y mejoramiento, basados en resultados cuantitativos que permitirán el análisis del comportamiento de dichos costos, frente a la toma de decisiones realizadas al respecto.

\section{LOS COSTOS DE CALIDAD Y LA NO CALIDAD Y LA UNIVERSIDAD EAN}

Como una de las entidades formadoras de directivos y administradores para el manejo de las empresas del país desde hace más de 40 años, la Universidad considera de importancia relevante la investigación e innovación en la gestión empresarial, como herramienta de aprendizaje y apoyo a la gestión de sus egresados y a las empresas para las cuales se han formado como expertos en administración o especialistas en áreas administrativas.
Dada la trascendencia de los costos de operación para la supervivencia de la empresa y el logro de sus objetivos sociales, los equipos altamente calificados que componen las áreas de investigación y docencia de la Universidad, directamente están abordando el problema de los costos de calidad y no calidad, con el fin de crear herramientas gerenciales de utilidad práctica para las empresas de salud y seguridad social, sector con una alta competencia y grave crisis financiera en muchas de sus empresas. 


\section{La CALidad TOtALSUS PRINCipios y LA IMPORTANCIA DE LOS COSTOS DE LA CALIDAD Y LA NO CALIDAD}

\begin{abstract}
Ua teoría sobre la calidad total en las empresas, desde los primeros autores considerados íconos a nivel mundial, reconoce la importancia del trabajo en calidad sobre los costos operacionales de la organización. Para apoyar la necesidad de calcular los costos de Calidad y No Calidad, se resumen a continuación algunos planteamientos:
\end{abstract}

- Deming, ${ }^{1}$ uno de los gurús de la calidad a nivel mundial, líder en el resurgimiento empresarial del Japón en la postguerra, establecía claramente que en la calidad lo importante es "Prevención, no corrección de errores" y en sus 14 principios para la implantación de la calidad, propuso que las empresas deben:

- Adoptar la nueva filosofía de conciencia de la calidad. Y a renglón seguido, reconoce la relación del trabajo en calidad con los costos de la empresa, cuando afirma que con la implantación de la calidad, en las empresas:

- Los costos disminuyen debido a menos reprocesos.

- Menor número de errores.
- Menos demora y obstáculos.

- Mejor utilización de las máquinas, del tiempo y de los materiales.

A su vez, Armand Feigenbaum, en sus pasos para la implantación del sistema de calidad, define como uno de sus 13 puntos: "Costo de calidad acompañado de otras mediciones y estándares de desempeño de la calidad".

Cada vez es más relevante la percepción de la calidad para los clientes y la supervivencia de la empresa, como lo afirma $\mathrm{H}$. James Harrington": "Ya no es posible sobrevivir con los niveles de defectos que aceptábamos antes."

Con unos clientes cada vez más enterados y una abierta e intensa competencia, la evidencia de fallas o problemas de calidad por parte de los clientes, fácilmente los induce a la búsqueda de servicios en la competencia. Como está bien comprobado en el mundo, el regreso de este cliente, es muchas veces más difícil y costoso que la consecución de uno nuevo. Las empresas de salud hasta hace muy poco, no se han preocupado por los costos de producción y mucho menos, han tenido herramientas administrativas para

\footnotetext{
${ }^{1}$ Deming, W.Edwards (1989).Calidad, productividad y competitividad. La salida de la crisis. Ediciones Diaz de Santos, S.A Madrid.

${ }^{2}$ Harrington, James; 1987, El Coste de la Mala Calidad, primera edición, Ediciones Díaz de Santos S.A.España.
} 
conocer los costos injustificados que les producen los errores y fallas en sus sistemas de producción, razón adicional para no preocuparse suficientemente de unos costos que están afectando significativamente sus utilidades y el margen de rentabilidad de la empresa. En un Sistema de Seguridad Social como el creado por la Ley 100 de 1993, los reducidos márgenes que permiten los techos financieros que se establecen para el aseguramiento y la prestación de los servicios de salud, obligan a las empresas a repensar en sus costos de producción y en contener aquellos no justificables.

Más adelante, Phillip Crosby establece en sus 13 pasos para la implantación del sistema de calidad, los siguientes como fundamentales:

- Medición.

- Costo de la calidad.

- Conciencia sobre la calidad.

- Eliminación de las causas de error.

Es decir, nuevamente resalta la relación entre la calidad y el costo.

Sin medir, es imposible saber la realidad de lo que está ocurriendo en las diferentes áreas y procesos de la empresa. ¿Cómo se pueden valorar los logros?, ¿Cómo evaluar el impacto de las decisiones de mejoramiento ó de contención de costos?
Todavía más específicos con los autores Alexander y Horngren cuando afirman:

"La calidad total se entiende como una estrategia competitiva que la alta gerencia decidió implantar para satisfacer mejor las necesidades de los clientes y aumentar el retorno de la inversión" (Alexander, 1994, pág. 1) Horngren, et al (1996, pág. 794) señalan que "se hace énfasis en la calidad, porque los costos asociados con la calidad son considerables. Los costos de calidad varían de 15 a $20 \%$ de los ingresos de ventas de muchas organizaciones, y con un buen programa de calidad, se pueden obtener ahorros sustanciales e ingresos más elevados".

Teniendo en cuenta que la medición de los costos de calidad y no calidad está basada en los procesos, es indispensable considerar algunos de los principios para el mejoramiento continuo establecidos por el Kaizen, filosofía de la calidad basada en el mejoramiento de los procesos, con el fin de eliminar los errores, optimizar la calidad y reducir los costos de producción en las empresas. Al respecto, es importante analizar algunos de las consideraciones de Masaaki Imai ${ }^{1}$ :

El kaizen es una metodología sistemática que se ha desarrollado con el fin de ayudar a una organización a realizar avances significativos en la manera de dirigir los procesos. El principal objetivo

\footnotetext{
${ }^{1}$ Imai, Masaaki. Cómo implementar el Kaizen en el sitio de trabajo (Gemba). McGraw Hill Interamericana. Bogotá,1998.
} 
consiste en garantizar que la compañía tenga procesos que:

- Eliminen los errores.

- Minimicen las demoras.

- Maximicen el uso de los activos.

- Promuevan el entendimiento.

- Sean fáciles de emplear.

- Sean amistosos con el cliente

- Sean adaptables a las necesidades cambiantes de los clientes.

- Proporcionen a la organización una ventaja competitiva.

- Hagan un uso más productivo del personal.

\subsection{Reducción de costos en el gemba (Unidades de Producción)}

Las oportunidades para la reducción de costos, donde sea posible, pueden expresarse en términos de muda (Desperdicio). La mejor manera para reducir costos en el gemba, es decir en la unidad o centro de producción, es eliminar el uso excesivo de recursos. Para reducir costos, las siete actividades siguientes deben llevarse a cabo en forma simultánea, siendo el mejoramiento de la calidad la más importante. Las otras seis actividades importantes de reducción de costos pueden considerarse como parte de la calidad del proceso, en un sentido amplio:

- Mejorar la calidad.

- Mejorar la productividad.

- Reducir el inventario.

- Acortar la línea de producción
- Reducir el tiempo ocioso de la maquinaria.

- Reducir el espacio.

- Reducir el tiempo del ciclo.

Estos esfuerzos para eliminar el muda (desperdicio) reducirán el costo general de las operaciones.

\subsection{Doce categorías de derroches}

- Sobreproducción.

- Existencias.

- Manipulación excesiva de los materiales.

- Esperas.

- Pérdidas de tiempo.

- Movimiento excesivo.

- Piezas defectuosas.

- Equipamiento defectuoso.

- Equilibrio en la carga de trabajo.

- Utilización incongruente de recursos.

- Diseño deficiente.

- Supervisión.

Como puede observarse, es evidente y constante en los tratadistas a nivel mundial de la calidad, la preocupación y relación de la calidad y los problemas de calidad con los costos operacionales de cualquier empresa.

Los errores cuestan dinero y este es tan considerable, que se considera relevante que las empresas conozcan el valor de las fallas en los procesos, con el fin de reducir los errores y los costos de los procesos respectivos. 
En este sentido, el kaizen propone estrategias para disminuir los desperdicios o moda, considerando los siguientes como los tipos de modas administrativas:

Duplicación de tareas, en parte por falta de información compartida

Exceso de movimientos y traslados, en parte como resultado de la mala disposición física

Excesiva subdivisión de procesos y/o actividades

Falta de trabajo en grupos o equipos de trabajo

Ineficiencia de control interno, lo cual ocasiona 4 tipos de problemas

Posibles fraudes externos contra la compañía

Posibles fraudes internos

Información poco confiable o inexacta a los efectos de la toma de decisiones

Falta de cumplimiento a normativas de organismos oficiales

Excesiva cantidad de formularios, con duplicación de información y/o innecesarios

Formularios mal diseñados

Excesivo inventario / stock de formularios

Tareas, procesos y $/ 0$ actividades innecesarios

Actividades o procesos complejos

Falta de información y administración por excepción

Listados de cómputos: innecesarios y/o mal diseñados

Información fuera de tiempo y/o inexacta

Sobre-estructura organizativa. Ello puede ser el resultado de excesivos actividades manuales, tramos de control demasiados cortos, falta de empowerment, carencia de objetivos organizacionales claramente definidos o política de sobre-empleo

Software: inadecuado, no parametrizable, de lenta ejecución, no adaptado a las características de la empresa o del negocio

Cuellos de botella originados en: concentración o centralización de decisiones o autorizaciones, cantidad de elementos críticos escasos en función de las necesidades-llámense: computadoras, impresoras, fotocopiadoras y procesos de cómputos

Averías y falta de mantenimiento en computadoras, impresoras, sistema lumínico, teléfonos

Falta de resguardo de datos informáticos

Deficiente supervisión

Deficiente capacitación del personal

Excesivos niveles de estandarización o normativas internas

Exceso de informes internos

Exceso de reuniones internas y/o interrupciones externas

Improductividades por exceso de especialización o división de trabajo

Lentitud de impresoras, fotocopiadoras o procesos de cómputos

Transcripción completa del texto del autor para mayor aporte conceptual a los lectores. 
La ineficiencia antes mencionada es razón por la que organizaciones vinculadas a la educación o la salud, pierdan gran parte de sus recursos, sino la mayor parte, en gastos administrativos aparte de los ilícitos que a través de estas actividades suelen llevarse acabo.

Los costes administrativos son elevadísimos; pueden representar hasta el $30 \%$ del coste total de una empresa o a veces más. Eso considerando sólo los costos explícitos. Los implícitos pueden llegar a ser más elevados, información errónea o fuera de término, falta de seguimiento y control, carencia de información para la toma de decisiones son entre otras las causantes de dolores de cabeza para los niveles gerenciales de una organización.

La oficina viene a ser el cerebro de la empresa. La mayoría de los directivos y profesionales, por no decir todos, controlan e influyen, o se ven afectados por el modo en que funciona la oficina. Pese a ello, pocas empresas disponen de un sistema de formación o de ayuda al directivo que tiene a su cargo la oficina. Los profesionales expertos en ingeniería, ciencias, contabilidad, derecho o cualquier otro campo de especialización, no reciben ayuda alguna en el aprendizaje sobre el modo óptimo de desempeñar las funciones de oficina.

El $30 \%$ de los costes de las empresas tiene su origen en las funciones de oficina, que son vitales para la empresa y comprenden la planificación, el control, la contratación de personal, las compras, la contabilidad, la financiación, las cuestiones jurídicas, y otras muchas actividades. Sin embargo, pese a las dimensiones y costes de dichas funciones, hasta el momento apenas se les ha prestado atención.

Hay que estar inmerso en el mundo de los negocios para conocer la profunda influencia que ejerce la oficina y comprender lo ineficaces que en ocasiones pueden ser los sistemas utilizados en ella.

Según un estudio realizado por Booz, Allen \& Hamilton, las empresas podrían lograr aumentar su productividad entre un 13 y un $18 \%$ mejorando el propio rendimiento de los empleados administrativos. Casi el $80 \%$ de los costes de oficina se deben a los directivos y profesionales, es decir, a los trabajadores intelectuales. El estudio averiguó que estos trabajadores sólo invierten un $8 \%$ de su tiempo en la realización de análisis; un $30 \%$ lo dedican a actividades "menos productivas"; entre un 40 y un $50 \%$ a reuniones, y un $15 \%$ a la elaboración de documentos.

La oficina no es un espacio físico; es, ante todo, una actividad. Se trata de la formación, tramitación, análisis y utilización del trabajo administrativo que sirve de apoyo a una empresa y que se lleva a cabo en cualquier lugar o departamento donde se realicen estas actividades. 
La mala calidad le cuesta a la economía de todo el país, como lo afirma Manuel García en su libro: "Cultura de la Calidad"1.

Del mismo autor tenemos las siguientes como algunas de las principales causales de costos de prevención, evaluación o control y fallas internas y externas. Se transcriben en esta revisión como las trata el autor, pero como puede observarse, se pueden describir fácilmente su equivalente en los procesos de las empresas de salud:

\subsection{Costo de prevención de la calidad}

Son todos los gastos realizados para evitar que se cometan errores, dicho de otra manera, es el dinero que se gasta para que los trabajadores hagan bien lo que tiene que hacer desde la primera vez. Visto esto financieramente, se encuentra dentro de los costos indirectos, costos fijos y costos variables. Se podría decir sin lugar a dudas que es una inversión a futuro. Se incluyen aquellas actividades de prevención y medición realizadas durante el ciclo de comercialización, son elementos específicos los siguientes:

\begin{tabular}{|l|}
\hline Revisión del diseño \\
\hline Calificación del producto \\
\hline Revisión de los planos \\
\hline Orientación de la ingeniería en función de la calidad \\
\hline Programas y planes de aseguramiento de la calidad \\
\hline Evaluación de proveedores \\
\hline Capacitación a proveedores sobre calidad \\
\hline Revisión de especificaciones \\
\hline Estudios sobre la capacidad y potencialidad de los procesos \\
\hline Entrenamiento para la operación \\
\hline Capacitación general para la calidad \\
\hline Desarrollo e implantación de un sistema de recolección y presentación de datos \\
\hline Auditorias y mantenimiento preventivo \\
\hline Auditorias internas \\
\hline Desarrollo del plan de control de calidad del proceso \\
\hline Estudio de proveedores \\
\hline Implantación del proceso de mejora continua \\
\hline Realización de revisiones del concepto de diseño \\
\hline Evitar que un problema se repita \\
\hline Planificación de la calidad \\
\hline
\end{tabular}




\begin{tabular}{|c|}
\hline Procedimientos de formación \\
\hline Contacto con los clientes para conocer sus expectativas \\
\hline Manuales técnicos \\
\hline Revisiones preproducción \\
\hline Actividades para la prevención de defectos \\
\hline Preparación de normas de trabajo \\
\hline Modelación y simulación de procesos \\
\hline Revisiones de requisitos \\
\hline Calificación del empaquetado \\
\hline Sistema de aseguramiento de la calidad \\
\hline Revisiones de las instalaciones \\
\hline Planificación de programas de cómputo \\
\hline Revisión de diagramas de flujo \\
\hline Análisis de correlación \\
\hline Plan de calidad del programa \\
\hline Plan del equipo de ensayos \\
\hline Análisis de fallos \\
\hline Estudios de capacidad de procesos \\
\hline Estudio de capacidad de máquinas \\
\hline Certificación de operarios \\
\hline Mantenimiento preventivo \\
\hline Revisiones del proceso \\
\hline Monitoreo y control ambiental \\
\hline Calificación de proveedores \\
\hline
\end{tabular}

Transcripción completa del texto del autor para mayor aporte conceptual a los lectores.

\subsection{Costos de evaluación de la calidad}

El costo de evaluación es el resultado de la evaluación de la producción ya terminada y la auditoria del proceso para medir la conformidad con los criterios y procedimientos preestablecidos. Mejor dicho, es todo lo gastado para terminar. 


\begin{tabular}{|c|}
\hline Auditorías de garantía de calidad del proceso de manufactura \\
\hline Auditorías financieras externas \\
\hline Auditorías internas y externas \\
\hline Inspección y ensayos para determinar la conformidad de los productos y/o \\
\hline Mantenimiento y calibración de equipos de ensayo e inspección \\
\hline Revisión de los diseños terminados \\
\hline Revisión de los datos de ensayo e inspección \\
\hline Repaso de los errores de las cartas \\
\hline Comprobaciones del comportamiento postventa \\
\hline Inspección y prueba de prototipos \\
\hline Análisis del cumplimiento con las especificaciones \\
\hline Vigilancia de proveedores \\
\hline Inspecciones y pruebas de recepción \\
\hline Actividades para la aceptación del producto \\
\hline Aceptación del control del proceso \\
\hline Inspección de embarque \\
\hline Estado de la medición y reportes de progreso \\
\hline Descripción del trabajo \\
\hline Materiales de ensayo e inspección \\
\hline Preparación para la inspección y ensayo \\
\hline Auditorías de calidad del producto \\
\hline Avales externos \\
\hline Valoración durante el proceso \\
\hline Controles de proceso \\
\hline Apoyo de la evaluación \\
\hline Registros de equipos de ensayo \\
\hline Administración del Dpto. de Calidad \\
\hline Formación del personal de calidad \\
\hline Costo de ensayos del sistema \\
\hline Revisión de facturación \\
\hline Auditorías de producto \\
\hline Auditorías de sistemas de calidad \\
\hline Auditoría de la satisfacción del cliente \\
\hline Evaluación por un laboratorio externo \\
\hline Ensayo de vida \\
\hline Análisis de envejecimiento y fatiga \\
\hline Prueba de inserción de fallos \\
\hline Análisis de verificación de códigos \\
\hline Verificación de estándares de trabajo \\
\hline
\end{tabular}

Transcripción completa del texto del autor para mayor aporte conceptual a los lectores. 


\subsection{Costos internos de la mala calidad}

Los costos internos de la mala calidad son todos los errores que tiene la empresa y que han sido detectados antes de que los bienes o servicios sean aceptados por el cliente, porque las actividades no se hicieron bien todas las veces.

\begin{tabular}{|l|}
\hline Costos de las fallas de los productos \\
\hline Costos de degradación del producto \\
\hline Horas extras debido a problemas \\
\hline Desechos o re-procesos \\
\hline Actividades de clasificación \\
\hline Reinspección a causa de rechazos \\
\hline Costo de la corrección de problemas \\
\hline Costo de la reinspección y los ensayos \\
\hline Acción correctora \\
\hline Informes de fallos \\
\hline Análisis de los desechos \\
\hline Análisis de los re-procesos \\
\hline Soporte de fallas \\
\hline Círculos de calidad \\
\hline Equipos de mejora \\
\hline Costo del rediseño y cambios de ingeniería \\
\hline Productos retirados \\
\hline Modificaciones del proceso \\
\hline Herramientas temporales \\
\hline Programas abandonados \\
\hline Actividades para reducir costos \\
\hline Costo de los errores de facturación \\
\hline Volumen de incobrables \\
\hline Costo de los errores de nomina \\
\hline Existencias no controladas \\
\hline Cuentas pendientes vencidas \\
\hline Pagos incorrectos a proveedores \\
\hline Revisiones del costo de la mala calidad \\
\hline Desechos del proveedor \\
\hline Re-procesos de las piezas del proveedor \\
\hline Accidentes \\
\hline Costo de morosos \\
\hline \\
\hline
\end{tabular}




\begin{tabular}{|l|}
\hline Robos \\
\hline Ausentismo \\
\hline Costo de la rotación de personal \\
\hline Retrasos \\
\hline Costos por no cumplir con el calendario \\
\hline Bienes y equipos estropeados \\
\hline Pérdidas de activos \\
\hline Transcripción completa del texto del autor para mayor aporte conceptual a los lectores. \\
\hline
\end{tabular}

Transcripción completa del texto del autor para mayor aporte conceptual a los lectores.

\subsection{Costos externos de mala calidad}

Los costos externos de la mala calidad son todos aquellos errores en que incurre el productor porque al cliente externo se le suministran productos o servicios inaceptables, entre los cuales tenemos:

\begin{tabular}{|c|}
\hline Cancelar proveedores \\
\hline Verificar fallo \\
\hline Centro de reparaciones de fallas \\
\hline Formación de personal para reparaciones de fallas \\
\hline Salarios para personal de reparaciones \\
\hline Perdidas de alquileres \\
\hline Cargos por tiempo improductivo \\
\hline Retirada de productos \\
\hline Costos y retrasos por modificaciones \\
\hline Escasez de componentes o materiales \\
\hline Servicio al producto a causa de errores \\
\hline Servicio al cliente por causa de errores \\
\hline Productos rechazados y devueltos \\
\hline Reparación de materiales devueltos \\
\hline Gastos de garantía \\
\hline Reinspección y repetición de ensayos \\
\hline Corrección d problemas \\
\hline Acciones correctoras \\
\hline Soporte por fallo de planta \\
\hline Desechos y reprocesos por cambios en ingeniería \\
\hline Análisis de las devoluciones \\
\hline Análisis de garantía \\
\hline Contacto directo con el cliente por problemas post venta \\
\hline Rediseño \\
\hline
\end{tabular}




\begin{tabular}{|l|}
\hline Análisis de los cambios de ingeniería \\
\hline Cambio de documentación \\
\hline Costo de morosos \\
\hline Informa de fallos \\
\hline Incobrables \\
\hline Robos \\
\hline Costos debido a esperas \\
\hline
\end{tabular}

Transcripción completa del texto del autor para mayor aporte conceptual a los lectores.

\section{Ventajas de LA MEdición DE LOS COSTOS DE CALIDAD Y NO CALIDAD}

- Es una herramienta de gestión que permite cuantificar en términos monetarios, la inversión realizada mes a mes en el mantenimiento y mejoramiento de la calidad y aquellos no justificados, producidos por fallas en los procesos (repeticiones, corrección de errores, reprocesos, repetición de atenciones).

- Se constituye en un apoyo y complemento al Sistema de Gestión Empresarial. Le aporta una base cuantitativa para la toma de decisiones dirigidas al mejoramiento de la calidad y a la contención de los costos de No Calidad

- Permite evaluar el impacto de la toma de decisiones, porque en forma cuantitativa al mes siguiente, muestra el valor de los costos de No Calidad de un determinado proceso, comparativamente con los meses anteriores. Se podrá analizar el impacto en reducción de los Costos de No Calidad obtenido luego de las decisiones de control establecidas en los meses anteriores.
- Permite comparar la inversión en calidad (Mantenimiento ymejoramiento de los procesos), frente a los costos de no calidad en cada proceso mes a mes. Obviamente, la inversión en calidad (Capacitación, inducción, reinducción, auditoría, control), debería reflejar una consecuente disminución en los costos injustificados por fallas en dichos procesos.

- Concientiza a la administración y a los empleados, en la necesidad de controlar los costos de operación y trabajar en el mejoramiento continuo de la calidad de los procesos. Cada mes en el Tablero de Costos de Calidad y No Calidad, se evidencian la inversión en calidad y los gastos injustificados producidos por las fallas y errores en cada proceso. Podrá cada responsable de proceso, participar activamente en el análisis de las causas y el establecimiento de correctivos y acciones de mejoramiento. 


\section{Conclusiones}

- La revisión bibliográfica analizada para el artículo, en forma clara evidencia la importancia del conocimiento de los costos invertidos en la calidad y los no justificados ó de no calidad generados por las fallas en los procesos, para mejorar la sostenibilidad financiera de la empresa, al contener apropiadamente los costos que no agregan valor a la empresa.

- Las empresas de salud por la multiplicidad de procesos, procedimientos y normas, entre otros factores, tienen una alta probabilidad de fallas 0 errores que producen unos costos injustificados que, de conocerse, permitirán a la empresa su reducción o control, mejorando su rentabilidad.

- La creación en las empresas de salud de sistemas de medición de costos de calidad y no calidad, permitirán conocer el monto de la inversión en la calidad de los procesos y el dinero perdido por costos de no calidad en forma sistemática y confiable, como soporte al Sistema de Gestión Empresarial.

\section{CAMBLAMOS!}

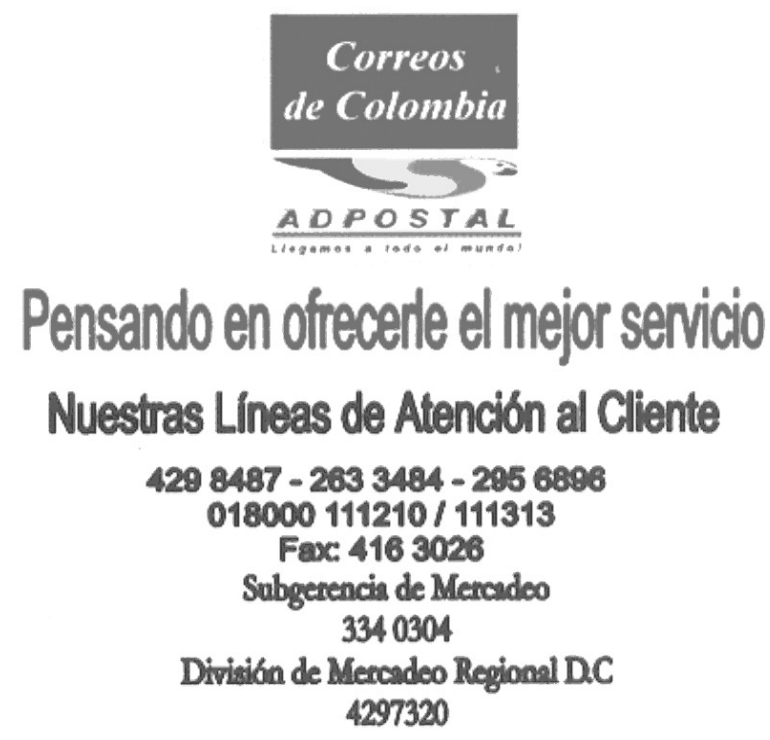

Www.adpostal.gov.co 


\section{Bibliografía}

Alexander, A. (1994): La mala calidad y su costo. Por Addison-Wesley. U.S.A. Iberoamericana. Wilmington, Delaware,

Barrie G., D. (1993): Los costos de la calidad. México. Primera Edición, Editorial Grupo Editorial Iberoamérica. México.

Besterfield, D. (1995): Control de calidad. México. Editoral Prentice Hall Hispanoamericana, S.A.

Campanenella, J. (1992): Principios de los costos de la calidad. Madrid, España. Ediciones Díaz de Santos, S.A.

Colunga Dávila C., Saldierna Gómez A. (1994). Los costos de calidad. Universidad Autónoma San Luis de Potosí. Facultad de Ingeniería.

Deming, W. E. (1989): Calidad, productividad y competitividad. La salida de la Crisis. Madrid. Ediciones Díaz de Santos, S.A.

García P., M. (1994): Cultura de la calidad. Lima Perú. Primera edición. Editorial UNMSM.

Harrington, J. (1987): El coste de la mala calidad. España. Primera edición.Ediciones Díaz de los Santos S.A.

Horngren, Ch; Foster, G. y Datar, S. (1996): Contabilidad de costos. Un Enfoque de Gerencia. México.Editorial Prentice Hall Hispanoamericana, S.A.

Imai, M. (1998): Cómo Implementar el Kaizen en el Sitio de Trabajo (Gemba).Bogotá. Editorial McGraw-Hill Interamericana, S.A.

Joseph M. J. (1990): Juran y la planificación para la calidad. Madrid, España. Ed. Díaz de Santos.

Naveh, E. y Halevy, A. (1996): El Costo de la No Calidad, 20(03). 